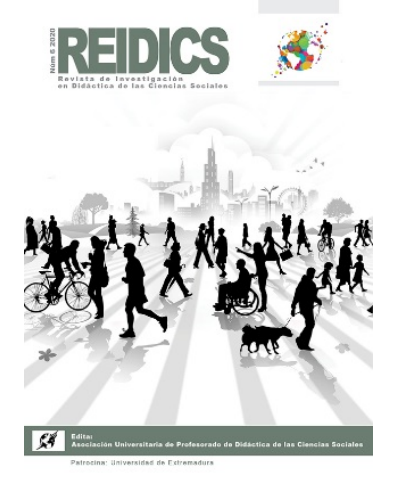

\title{
REIDICS
}

Revista de Investigación en

Núm. 7, 2020

Didáctica de las Ciencias

Recibido 30 de junio de 2020

Sociales

Aceptado 11 de agosto de 2020

E-ISSN: 2531-0968

\section{Uso de las redes sociales entre los jóvenes y ciudadanía digital: análisis tras la COVID-19}

Use of social media among young people and digital citizenship: analysis after COVID-19

\author{
Rocío González-Andrío Jiménez \\ Universidad Rey Juan Carlos de Madrid \\ Email: rocio.gonzalezandrio@urjc.es \\ ORCID: https://orcid.org/0000-0001-9548-8210
}

César Bernal Bravo

Universidad Rey Juan Carlos de Madrid

Email: cesar.bernal@urjc.es

ORCID: https://orcid.org/0000-0002-2802-1618

Irene Magdalena Palomero Ilardia

Universidad Rey Juan Carlos de Madrid

Email: irene.palomero@urjc.es

ORCID: https://orcid.org/0000-0001-7197-7431

DOI: https://doi.org/10.17398/2531-0968.07.64

\section{Resumen}

Este artículo se enmarca en un proyecto aprobado y financiado por el Consejo de Investigación de Ciencias Sociales y Humanidades de Canadá, sobre redes sociales, participación ciudadana y educación. El objetivo es explorar y comprender los impactos del uso, difusión y producción de las redes sociales en la participación ciudadana y la democracia. El nuestro es un estudio de tipo interpretativo, descriptivo del comportamiento de los jóvenes y corresponde a su fase inicial. Un cuestionario online ha sido respondido por 127 estudiantes, de los cuales el 95,05\% son españoles. En este trabajo se presentan los resultados más significativos cuantitativos y cualitativos, así como las cuestiones críticas en base a los resultados obtenidos con la herramienta ATLAS-ti para identificar pautas relevantes y establecer un marco que ofrezca un significado a las respuestas. Se concluye que los problemas a los que hacen más referencia los jóvenes encuestados en relación al uso de las redes sociales son los problemas éticos, entre los que destacan el engaño, la crueldad, el ciberbullying, el uso de las redes para descalificar o desacreditar a personas, la competitividad, las faltas de respeto a las opiniones ajenas, los juicios infundados y la existencia de contenidos "oscuros", lo que nos induce a cuestionarnos 
de qué modo afecta positiva o negativamente este hecho a su comprensión de la "participación ciudadana".

Palabras clave: educación ciudadana; redes sociales; democracia; jóvenes; Covid19

\begin{abstract}
This article is part of a project approved and funded by the Research Council of Social Sciences and Humanities of Canada, on social media, citizen participation and education. The objective is to explore and understand the impacts of the use, dissemination and production of social networks on citizen participation and democracy. Ours is an interpretive, descriptive study of the behavior of young people and corresponds to its initial phase. An online questionnaire has been answered by 127 students, of whom 95,05\% are Spanish. This paper presents the most significant qualitative and quantitative results, as well as the critical issues based on the results obtained with the ATLAS-ti tool, to identify relevant guidelines and establish a framework that offers a meaning to the answers, and concludes that the problems to which young people surveyed make more references in relation to the use of social networks are the ethical problems for instance: the deception, cruelty, cyberbullying, the use of networks to disqualify or discredit people, competitiveness, disrespect for other people's opinions, unfounded judgements and the existence of "dark" content, which leads us to question how this fact positively or negatively affects their understanding of "citizen participation".
\end{abstract}

Keywords: citizen education; social media; democracy; young; Covid19

\title{
1. Introducción
}

Este artículo se enmarca en un proyecto aprobado y financiado por el Consejo de Investigación de Ciencias Sociales y Humanidades de Canadá, sobre redes sociales, participación ciudadana y educación. El objetivo es explorar y comprender los impactos del uso, difusión y producción de las redes sociales (RS) en la participación ciudadana y la democracia en los jóvenes universitarios.

Internet ya es parte de nuestras vidas, supone una nueva forma de relacionarnos con la información, el conocimiento y las personas. Una de las respuestas a la pregunta “ ¿Qué piensa que va a suceder dentro de cincuenta años con Internet?" realizada a algunos expertos entrevistados desde Pew Research Center ${ }^{1}$ (PRC), nos anuncia un mundo en el que la vida sin internet no es posible. Ashok Goel, director de Human-Center Computing Ph. D. program at Georgia Tech, emplea tres adjetivos para referirse a este hecho: omnipresente, omnisciente y casi omnipotente Internet. De tal modo que, "everyone in the world will have access to the internet and the internet will have access to everyone and almost everything." 2

De acuerdo con internetworldstats.com³ ${ }^{3}$, España se encuentra en la segunda posición $(92,1 \%)$ entre los países de habla hispana en cuanto al número de usuarios de internet. Argentina se posiciona en primer lugar con un $93,1 \%$ de internautas. La cifra de personas con perfiles en RS

1 PRC is a nonpartisan fact tank that informs the public about trends shaping the world. Recuperado de https://www.pewresearch.org/about/U.C.28/06/2020

2 Recuperado de https://www.pewresearch.org/internet/2019/10/28/2-internet-pioneers-imagine-the-next-50years/U.C. 28/06/20

3 Recuperado de https://www.internetworldstats.com/stats13.htm U.C. 26/7/2020 
ha aumentado en el mundo en un 9\%, respecto al 2019, lo que supone 321 millones de usuarios más que el año anterior. ${ }^{4}$

La encuesta realizada por el Instituto Nacional de Estadística (INE) en España en el año 2019 sobre el uso de Internet, señala que está extendido sobre todo entre los jóvenes de 16 y 24 años. Un $99,2 \%$ son hombres y un $99,0 \%$ son mujeres ${ }^{5}$. Por tanto, podemos afirmar que los jóvenes son los "dueños" del ciberespacio.

Los estudiantes acceden habitualmente a Internet a través de los móviles de última generación. El uso ininterrumpido del móvil se ha extendido hasta tal punto, que los problemas de ciberadicción son realmente preocupantes (Billieux, Maurage, Lopez-Fernandez, Kuss, \& Griffiths, 2015).

\section{Investigación}

Este estudio se acomete desde la perspectiva de los recientes acontecimientos que han provocado la mayor crisis de nuestro siglo. Una epidemia viral ha trasformado de la noche a la mañana a todos los habitantes del planeta en ciudadanos aún más dependientes de Internet y seguidores de las RS. "In December, 2019, an outbreak of a novel coronavirus (...) started in Wuhan, China, and has since become a global threat to human health" (Zhang, Shi, \& Wang, 2020, p. 428).

Durante el estado de alarma y el confinamiento6, el mundo de la cultura, cantantes y deportistas, entre otros, han ofrecido a los ciudadanos y ciudadanas del mundo entero el acceso a bibliotecas virtuales, a conciertos y eventos deportivos en línea, gracias a las RS. Cada una de estas iniciativas son ejemplos gráficos de nuevos modos de participación ciudadana online. Pero, al mismo tiempo las redes han sido el canal de comunicación utilizado para difundir desinformación, mentiras o los llamados "bulos" aun cuando la verdad se ha convertido en un tema de vida o muerte (Pennycook, McPhetres, Zhang, \& Rand, 2020).

En este contexto en el que los interrogantes sobre el futuro de Internet se multiplican, nos preguntamos de qué modo pueden afectar positiva o negativamente los razonamientos juveniles y modo de entender las RS a su comprensión de la "participación ciudadana digital". Las respuestas obtenidas nos llevan a reflexionar asimismo sobre el reto educativo que tenemos por delante.

Nos preguntamos sobre los nuevos paradigmas éticos online. “¿En qué nos convertimos a medida que los cambios tecnológicos van alterando el paisaje material y social?" (Bustamante Donas, 2007, p. 206) o en qué nos queremos convertir.

\footnotetext{
4 Recuperado de https://mcsocialmedia.com/estadisticas-redes-sociales-usuarios-activos-facebook-instagramyoutube-twitter-linkedin-otras/ U. C. 28/06/2020

5 Recuperado de https://ine.es/prensa/tich_2019.pdf. U.C. 28/06/2020

6 Recuperado https://www.lamoncloa.gob.es/consejodeministros/resumenes/Paginas/2020/14032020_alarma.aspx U. C. $28 / 06 / 2020$
} 


\section{La participación ciudadana de los jóvenes en la era digital: nuevos retos en la educación cívica}

Actualmente contamos en el mundo con la generación de jóvenes más numerosa de la historia. La cifra asciende a 1.800 millones de personas, de las cuales cerca del $90 \%$ viven en países en desarrollo ${ }^{7}$. Recordemos que fueron los jóvenes con la ayuda de las RS los que promovieron en el año 2010 un modo diferente de participación ciudadana, la llamada "Primavera Árabe", en la que una serie de manifestaciones ciudadanas reclamaban derechos sociales, "in an era where political action can come in the form of a social media movement, it is important to realize that the Internet has rewritten the rules not just of communication, but of citizenship as well" (Nelson, Lewis, \& Lei, 2017, p. 4).

Las cuestiones relacionadas con la participación juvenil y el concepto de ciudadanía se debaten desde hace años. Algunas de las principales conclusiones de esta discusión se pueden resumir del modo siguiente:

En primer lugar, nos encontramos con la creencia de una posible falta de compromiso ciudadano juvenil. "Undoubtedly many young citizens have indeed become disenchanted with mainstream political parties and with those who claim to speak on their behalf." (Loader, Vromen, \& Xenos, 2014, p. 143). Sin ciudadanos que participen a nivel local o global, la democracia pierde sentido, "democracy needs a participatory culture. People are expected to be interested in their citizenship responsibilities" (Simsek \& Simsek, 2013, p. 132).

Si la participación ciudadana es la piedra angular de la democracia, se necesita que los ciudadanos tomemos parte en los asuntos a nivel local y global de manera libre, e informada. Sin dicha participación la democracia se iría fragmentando progresivamente. El escaso interés en tomar parte en temas políticos, con el paso del tiempo, es considerado un factor clave para que se dé una crisis participativa de mayor envergadura (Castellanos Claramunt, 2019). Pero el debate sobre la necesidad de una educación ciudadana hunde sus raíces desde hace tiempo en temas más conflictivos de los señalados anteriormente. Entre otros, se reconoce la tiranía de lo políticamente correcto o lo que algunos autores llaman la práctica de la virtud cívica (Kymlicka \& Norman, 2000).

En segundo lugar, los regímenes políticos en que se desarrollan las RS suelen responder a lo que hoy se denomina "democracia" y no hay que olvidar que, para que esta sea real, ha de someterse continuamente a una reflexión y consiguiente revisión que adecúe realmente el orden político a los cambios de circunstancias que genera de continuo la vida social, como, por lo demás, observa (Rodríguez, 2008).

El momento de la historia en el que vivimos, abordar de nuevo el tema de la educación para la ciudadanía no es tarea fácil, ya que la noción de ciudadanía sigue teniendo significados variados, potencialmente diferentes e incluso contradictorios (Levinson, 2014; Rodríguez, 2008).

\footnotetext{
7 Recuperado de https://www.un.org/youthenvoy/wp-content/uploads/2014/09/UN-Youth-Strategy-Spanish1.pdf U.C. 28/06/2020
} 
Si el término de ciudadanía sigue siendo ambiguo, el debate sobre una educación ciudadana digital y global es algo complejo de abordar, más cuando el concepto de ciudadanía está marcado por los virajes ideológicos. (Delgado-Algarra, Bernal-Bravo, \& López-Meneses, 2019)

Por otro lado, el reto que ofrece la nueva era digital, los cambios e incertidumbres que se suceden a gran velocidad, son para los profesionales de la enseñanza una ocasión más para hacer una reflexión ponderada sobre lo que es o no la ciudadanía y la ciudadanía digital, siendo conscientes que "(...) cada persona porta su propia "brújula temporal” que les permite estructurar su pensamiento y su accionar."(Cartes., 2020, p. 9)

Las redes sociales no son algo neutro. Nos encontramos pues ante el interrogante sobre el futuro de la educación cívica digital, "the impact that these new tools may have on the future of online politics will largely depend on how millennials behave" (Calzada, 2017, p. 13).

Lo más llamativo es que todo sucede a una velocidad inimaginable.

Y nadie sabe bien lo que pasa antes de poder contar lo que pasa. Por eso, la contemporaneidad es un logro que requiere que nos demos una interpretación, que arriesguemos una visión de lo que ocurre, que nos permita tomar posición libre, pero justificadamente (Marín, 2019, p. 12).

Son dos los puntos que pueden dificultar el reto educativo de ciudadanos digitales:

1. La ausencia de un acuerdo común a nivel global en incluir la educación del pensamiento crítico en los programas oficiales, y la de un profesorado capacitado, que lleve a cabo un cometido de semejante envergadura (Mata, Sabater, \& Blanch, 2019).

2. La falta de un acuerdo universal sobre cómo debemos actuar en relación con las tecnologías digitales, "Will reaching an agreement be easy? Quite the opposite; it will be very difficult to come to a consensus on how everyone will deal with digital technology" (Ribble, Bailey, \& Ross, 2004, p. 11). Entendemos que, "even in a connected world, it takes time, effort and willpower to get major transformative effects from new technologies"(Fitzgerald, Kruschwitz, Bonnet, \& Welch, 2014, p. 4).

Trabajamos con una tecnología de innegable potencial, en el que la inmediatez y las posibilidades de interconexión e intercambio de ideas a nivel cultural son infinitas, pero sobre todo es un espacio ilimitado de expresión de la sociedad civil (Castells, 2003).

Cuando se entiende el poder que los jóvenes pueden llegar a tener en sus manos como ciudadanos activos con el uso que hacen de las RS, una de las cuestiones vinculadas con la participación ciudadana y con los derechos a repensar, es cómo éstos se pueden o deben instaurar de un modo adecuado y en un espacio de tiempo relativamente corto en una sociedad, en una cultura y sistema político (Heater, 2007).

\subsection{La ciudadanía digital y los nuevos espacios de participación social}

Con el término 'ciudadanía digital' nos referimos a todos aquellos internautas que, desde cualquier dispositivo válido con acceso a internet, interactúan, se comunican, comparten y reciben información, colaboran con la opinión pública u ofrecen ayuda o material sanitario, como ha sucedido durante la pandemia COVID-19. Sin embargo, la brecha digital en España y en otros 
muchos países del mundo, ha impactado directamente en la educación, entre los que carecen de recursos tecnológicos.

Desde el nacimiento de las primeras llamadas "social media" se decía que las redes iban a modificar nuestro modo de relación social, pero sobre todo que iban a suponer una drástica aculturación que afectaría a distintas esferas de la persona humana (Padilla Castillo, 2013).

Las tecnologías nos han influido en nuestro estilo de vida, pero, a la par de sus beneficios, cada día aparecen nuevos prejuicios que invitan a preguntarnos por los desafíos y retos que propone esta era de tecnología en materia de educación, relaciones sociales y salud ante el reto de formar a un ser humano que continúa a la expectativa de significar y controlar lo que existe a su alrededor. (Nobles et al., 2015, p. 15)

El mundo digital y las redes progresan día a día e incorporan mejoras para sacar el máximo partido a su uso. Los jóvenes se van adaptando a ellas con gran facilidad, dejando atrás las redes obsoletas y evolucionan de acuerdo a su edad y madurez humana. Los estudiantes van incorporando a sus vidas las nuevas redes que aparecen en el mercado y lo hacen de acuerdo con las posibilidades que éstas les ofrecen y a sus intereses individuales. (Mercedes, 2015).

El centro de investigación americano (PRC) plantea un tema interesante acerca de las nuevas tecnologías. Cuestiona si el uso de la tecnología por parte de los seres humanos debilitará la democracia a corto y medio plazo, señalando como causas principales la velocidad y el alcance que tiene la distorsión de la realidad, la disminución del periodismo y el impacto del capitalismo de vigilancia o si por el contrario, la tecnología fortalecerá la democracia a medida que los reformistas encuentran la manera de luchar contra los "infoguerreros" y el caos. 8

Con el uso habitual de las RS se ha extendido el uso del término e-democracia como una nueva forma de participación digital en la que el ciudadano de "a pie" opina, habla, comenta o critica lo que le parece debe ser corregido o modificado.

La opinión pública está formada desde hace muchos años, no solo por los periodistas sino por todos los ciudadanos y ciudadanas, de tal manera que, "for some students, participation is a process in which all the individuals in a society share the same goal and participate towards its achievement" (Sant, 2014, p. 18). Un claro ejemplo son las recientes manifestaciones ciudadanas a nivel global por la lucha del cambio climático.

\subsection{La nueva dimensión educativa y ética en el uso de las redes sociales en internet}

Los acontecimientos pasados y presentes nos llevan a repensar el futuro de la educación y la necesidad de profesionales más preparados, que no solo manejen Internet y las RS. Es vital conocer y entender a un alumnado que vive en su espacio virtual.

Entramos en el terreno de la "nueva dimensión ética" que tiene su origen en la era digital y global. Una ética que ya no solo afecta a la esfera pública offline, sino al ciberespacio público y privado online. La libertad de expresión es compatible con el respeto a los derechos individuales y colectivos, con el ejercicio de esos mismos derechos y el cumplimiento de las obligaciones fuera

8 Recuperado de https://www.pewresearch.org/internet/2020/02/21/many-tech-experts-say-digital-disruptionwill-hurt-democrac/ U.C. 28/06/2020 UC 28/6/2020 
y dentro de Internet. Aprender a ser una ciudadana o un ciudadano forma parte de la educación, como es el aprendizaje a ser persona y un agente moral en una comunidad. (Naval, 2003). Por tanto, "introducir la tecnología en este contexto ético significa traducir el discurso ético en términos que engloben a la ciencia y a la tecnología en el espacio en el que se manifiestan, profundizan, y desarrollan los derechos humanos" (Bustamante Donas, 2007, p. 306).

\section{Objetivos de la investigación}

El primer objetivo es explorar y comprender los impactos del uso, difusión y producción de las redes sociales en la participación ciudadana juvenil y la democracia. Se trata de recuperar la voz de los jóvenes protagonistas del siglo XXI, de cara a un posible debate académico sobre educación y participación ciudadana digital.

Este objetivo se desglosa en los siguientes puntos:

- Conocer qué uso hacen de las redes sociales los jóvenes universitarios.

- Presentar el tipo de participación y compromiso ciudadano que llevan a cabo a través de las redes sociales.

- Exponer y analizar los asuntos más críticos que emergen de las respuestas obtenidas en cuanto a los pros y los contras del uso de las redes sociales.

- Identificar qué ayuda reciben los jóvenes desde el ámbito académico para llegar a ser ciudadanos/as digitales comprometidos.

- Determinar el tipo de formación que creen que necesitan los jóvenes en su uso de las redes sociales y la participación ciudadana.

El segundo objetivo nos llevará a reflexionar sobre los resultados obtenidos y examinar de qué modo afecta positiva o negativamente el uso de las redes sociales a su comprensión de la "participación ciudadana".

El tercer objetivo es presentar los retos educativos que tenemos por delante para formar una ciudadanía que participa y se expresa de modo virtual.

\section{Diseño y metodología}

Este estudio descriptivo e interpretativo ha utilizado como método de recogida de información una encuesta online anónima basado en otros estudios. ${ }^{9}$ La muestra estuvo compuesta por 127 estudiantes. El 87\% pertenecen a los grados de Educación Primaria e Infantil. El cuestionario original se presentó a cinco expertos para analizar la adaptación del contenido a la cultura española y ser validado. Se ha utilizado la prueba no paramétrica (W) de Kendall para probar el acuerdo de los expertos con un valor de 0,90. La fiabilidad con Alfa de Cronbach es de 0,89 con un error muestral del $5 \%$.

\footnotetext{
9 Hoechsmann, Michael, Thésée, Gina y Carr, Paul R. (eds.). (In press / À paraître). Education for Democracy 2.0: Changing Frames of Media Literacy. Rotterdam: Brill/Sense. Carr, Paul R., Hoechsmann, Michael y Thésée, Gina. (eds.). (2018). Democracy 2.0: Media, Political Literacy and Critical Engagement. Rotterdam: Brill/Sense. Carr, Paul R, Cuervo, Sandra y Daros, Michelli. (2019). Citizen engagement in the contemporary era of fake news: Hegemonic distraction or control of the social media context? Postdigital Science and Education, 1-22. Recuperado de https://doi.org/10.1007/s42438-019-00052-z U. C. 28/06/2020 y otros.
} 
La encuesta consta de 10 ítems de información sociodemográfica y 24 ítems sobre RS. Las preguntas de la sección de las RS son de respuesta escala Likert que nos permiten medir actitudes y conocer el grado de conformidad de los encuestados respecto a las preguntas planteadas. De este modo conocemos la intensidad de la afirmación respecto al nivel de importancia, frecuencia con la que se realiza una actividad, y valoración de un ítem o nivel de acuerdo. A estos datos cuantitativos se añaden las preguntas abiertas en las que los estudiantes pueden expresar con palabras lo que piensan respecto a cada cuestión.

El cuestionario original fue modificado ligeramente para ser adaptado al lenguaje y a la cultura española. De este modo se añadió la referencia a la LOPD. Se incluyeron los datos personales de los nuevos investigadores. Se añadió un desplegable para elegir la Universidad en la que estudian. Se añadió al curso la opción de Master. Se incorporó un nuevo desplegable con más áreas de conocimiento. En cuanto a los miembros de la familia que tienen estudios universitarios se añadió a cada ítem de la generación: 'solo yo', 'padres', 'abuelo' o 'bisabuelos'. A la pregunta del tipo de compromiso se pidió que se especificaran los niveles de participación. Se añadieron redes como: Tik Tok. Change.org WhatsApp y 21 buttoms. En cuanto al número de horas del uso se incluyó más de seis. Se cambió 'Presión social de las parejas' por 'Comunicación con la pareja/familia'. Se añadieron otras áreas de interés participativo como el de los Derechos Humanos.

\section{Resultados de la investigación de tipo cuantitativo y cualitativo}

\subsection{Datos de interés general sobre los participantes y uso de las redes sociales $(Q 3,5,8,9,13,14,15$ y16) 10}

El $87 \%$ de los participantes pertenecen a los grados de educación Primaria e Infantil en español y bilingüe. Al ser grados en los que domina el sexo femenino sobre el masculino, el 81,10\% de las respuestas corresponden a mujeres. ${ }^{11}$ frente al 18,10\% que son hombres. El 65,35\% de los encuestados pertenece a la primera generación de su familia con estudios superiores.

El 58,27\% lleva más de siete años usando las RS siendo las más utilizadas por este orden: WhatsApp, seguida de Instagram, Facebook y YouTube. El promedio de tiempo de uso de las redes es de entre tres y cuatro horas al día. Utilizan las redes para comunicarse con amigos/as (59,06\%) y con la pareja $(20,47 \%)$. También las utilizan como entretenimiento, para compartir opiniones o para comunicarse con miembros de la familia. Los jóvenes prefieren comunicarse en primer lugar con texto $(62,99 \%)$ seguido de audio $(21,26 \%)$ e imágenes $(12,60 \%)$.

En relación con la evolución que han seguido en las RS los encuestados explican que éstas han pasado de ser un mero entretenimiento, en su etapa inicial, para llegar a jugar un papel más útil o profesional, con un uso más prudente, equilibrado y responsable. Asimismo, los estudiantes

10 Q: número de pregunta del cuestionario.

11Recuperado de https://www.eldiario.es/sociedad/grados-paritarios-informatica-hombreseducacion_1_1735777.html U.C. 28/06/2020 
aumentan el uso de las RS y cambian de RS de acuerdo con la etapa madurativa, la evolución o las mejoras que incorporan las redes.

\subsection{Razones por las que utilizan las redes sociales ( $Q$ 16.4 y 17)}

Entre los jóvenes domina el uso personal de las RS frente al educativo o cultural. Los participantes enumeran varias razones por las que utilizan las RS: la facilidad para comunicarse entre ellos, la pareja o familia, compartir fotos, conocimiento e inquietudes sociales. Por último, señalan que las utilizan para organizar trabajos de clase, para el entretenimiento o como canal de promoción profesional.

Un dato interesante que queremos mencionar es que de entre los medios tradicionales de comunicación, son los telediarios los más vistos y considerados los más imparciales. Los periódicos impresos son desde el punto de vista de los estudiantes los más veraces.

\subsection{Uso que hacen los jóvenes de las redes sociales y temas por los que manifiestan más interés (Q11)}

El 84,13\% de los estudiantes están interesados en temas relacionados con la educación. El $11,90 \%$ destaca una preocupación por el medioambiente. El 2,36\% manifiestan interés por los partidos políticos. El 1,59\% por temas relacionados con la salud. En relación con el ocio y el entretenimiento, les interesa en primer lugar la música seguido de la moda, la fotografía, el cine, el arte y los libros. También utilizan las redes para buscar destinos turísticos, organizar viajes o acceder a información sobre temas de medio ambiente.

Los estudiantes manifiestan interés en primer lugar por las desigualdades sociales; seguido de la falta de equidad de género, derechos humanos, cuestiones raciales y culturales.

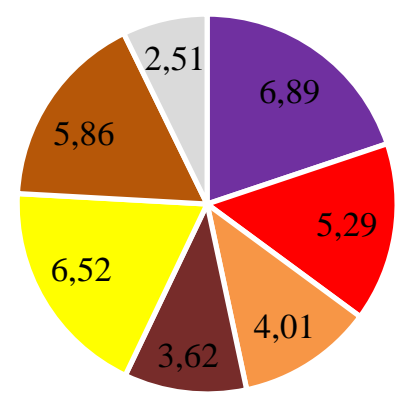

\footnotetext{
- Desiguladades sociales - Desiguladades sociales

- Tema raciales y culturales - Cuestiones económicas

Cuestiones políticas - Derecho Humanos

Otros temas
}

Figura 1. Temas con los que los estudiantes se sienten más vinculados (\%)

Fuente: Elaboración propia. 


\subsection{Tipo de participación y compromiso social que llevan a cabo a través de las redes los jóvenes universitarios ( $Q 10$ Y 12)}

El total de los estudiantes que se consideran miembros socialmente responsables es de un $69,05 \%$. Un 15,08\% no participa en la mejora de su comunidad y otro $12,70 \%$ se considera un miembro crítico al analizar las causas de los problemas y actuar sobre las mismas. El 3,17\% se reconoce un miembro activo al organizar únicamente campañas o recoger firmas para diversas propuestas de tipo social. Un estudiante no contesta.

A la pregunta sobre el tipo de participación que llevan a cabo en las redes el 51,91\% contestan que no tiene compromiso de tipo político.

Tabla 1

Tipo de participación ciudadana juvenil en \%

\begin{tabular}{ll}
\hline Tipo de participación & $\%$ \\
\hline Estudiantil - delegado de clase facultad & 5,51 \\
Asociaciones de estudiantes & 1,57 \\
Asociación de vecinos & 0,00 \\
Voluntariado: cruz roja, protección civil, organizaciones deportivas, prisiones, & 16,54 \\
culturales, ocio etc. & \\
ONG, fundaciones, asociaciones de diversa índole, infancia, personas mayores, & 14,17 \\
enfermedades, educativas, tiempo libre, laborales, consumidores etc. & \\
Partidos políticos & 2,36 \\
Sindicatos & 0,79 \\
No hay compromiso en la política & 55,91 \\
\hline
\end{tabular}

Fuente: Elaboración propia.

\subsection{Compromiso y participación ciudadana de los universitarios en los medios sociales $(\mathrm{Q} 24.12)$}

Los encuestados que contestan de modo positivo a la pregunta sobre el tipo de influencia que ejercen las RS en su compromiso social razonan diciendo que las redes son un medio óptimo para influir en las personas y para mejorar el mundo. El ejemplo más nombrado es la ayuda que ha supuesto Internet para la lucha por el cambio climático.

Los jóvenes explican que las redes facilitan el compromiso ciudadano y una participación más activa en la sociedad, por ser un canal de comunicación que puede influir de modo favorable en la manera de pensar, en las actitudes e intereses de las personas. Otros motivos mencionados son: porque se puede compartir todo, difundir ideas que pueden favorecer la reflexión, el debate o la concienciación social, facilita el estar al día sobre las noticias o porque ayuda a una buena convivencia social.

Un solo participante dice que cuando se «politizan» las RS no ayudan al compromiso social.

Otras ventajas señaladas por los universitarios en el uso de las RS se pueden resumir en:

- Facilitan el acceso a la información local y global.

- Ofrecen oportunidades de acciones solidarias.

- Incrementa la participación ciudadana activa en general. 
- Favorece la interconexión global. Por ejemplo alerta sobre los movimientos o manifestaciones sociales.

- Anima al dialogo y debate ciudadano.

- Ofrece gran visibilidad en temas sociales que animan a participar de forma presencial.

- En las redes se conecta rápidamente con las ciudadanas/os.

- Con un solo perfil se tiene acceso a gran variedad de información.

Una de las participantes señala que:

"En la medida que se demuestra seriedad y compromiso en la red, se puede favorecer la participación ciudadana".

\subsection{La influencia de las redes sociales en la democracia (Q36. 24)}

Algunos jóvenes señalan que los políticos pueden acercarse más al pueblo a través de las redes en la medida que éstos intervienen en ellas de un modo imparcial y veraz. Entienden que las redes son un canal de comunicación que facilita la libertad de expresión y ofrecen a los ciudadanos/as nuevas posibilidades para involucrarse desde el punto de vista social, para compartir, participar y reflexionar sobre política. Todos los jóvenes están de acuerdo en que son un medio perfecto para obtener información a nivel local y mundial.

Otros estudiantes explican que cuando las RS se utilizan para informar a la sociedad de una manera imparcial, poco equilibrada o injusta, el efecto en los ciudadanos/as es negativo. De igual manera, entienden que la existencia de contenidos no veraces en la red, actitudes y comportamientos extremistas, imposición de ideas o la existencia de diferentes modos de manipular a los ciudadanos a través de las redes, no beneficia a la democracia.

Otros participantes expresan otros motivos por los que la e-democracia puede deteriorarse. Explican que el subjetivismo en la transmisión de información online o el comportamiento poco ejemplar de los políticos que utilizan las redes para crear polémica o "promocionase", tampoco ayuda a la democracia.

Una de las estudiantes añade:

"Si las redes son utilizadas para difundir falsedades sobre temas económicos o sociales, el efecto sobre la democracia no es positivo". "No puede haber verdadera democracia si las redes son controladas por el gobierno." "Si las redes benefician a ricos y no a la clase obrera, eso no es democracia".

\subsection{Pros y contras en el uso de las redes sociales (Q16.4, 17.5 y 20.8)}

Los problemas en el uso de las RS enumerados por los estudiantes por orden de importancia son:

1. Problemas de tipo ético en la utilización de las redes

2. Problemas relacionados con la privacidad personal.

3. Problemas con la ciberadicción y la ausencia de un trato personal presencial

4. El ciberbullying y ciberseguridad.

Uno de los participantes explica que: 
"Un mal uso de las redes sociales por parte de los ciudadanos puede perjudicar en gran medida la educación de los jóvenes".

Aunque algunos estudiantes no llegan a detallar qué entienden por un mal uso de las RS, otros lo unen a la 'falsedad' y al uso de las redes para 'fines negativos, o hacen referencia al peligro del anonimato en la red o a la existencia de falsos perfiles con el riesgo de llevar vidas ficticias o dobles vidas. Los jóvenes entienden que ser uno mismo/a en la vida real y en la virtual es lo correcto.

Una de las participantes explica:

"Muchos jóvenes se sienten respaldados tras perfiles falsos o anónimos para realizar o decir cosas que no harían de saberse sus nombres. Lo hacen para aparentar algo que no son y jamás se podrá saber la verdad"

En cuanto a la información que se encuentran en las redes explican, que la ingente cantidad de contenidos en Internet es algo positivo porque es su fuente principal de conocimiento. Sin embargo, reconocen que es difícil contrastar la información.

El 51,20\% reconoce haber visto noticias falsas, el 24\% regularmente y al 79\% les preocupa el tema de la información errónea. Explican que el motivo principal es porque las noticias se hacen "virales" y la rectificación no tiene el mismo efecto o es difícil contrastarla con la verdad. Una participante dice que "Los estudiantes más influenciables e ingenuos son los más vulnerables y los que están más expuestos al peligro en las redes".

Otra participante explica:

"Es fácil aceptar noticias falsas sobre derechos humanos, política, racismo o machismo. Creo que se da mucho. Esto lleva a la confusión y conflictos sociales o sentimientos de odio." "La difusión de temas falsos sobre salud o relacionados con la alimentación, nos afecta negativamente".

A los encuestados que no les preocupan las noticias falsas lo razonan diciendo que si les interesa un tema se informan y lo contrastan.

En cuanto al grado de satisfacción que tienen de la imagen personal que proyectan en las redes (Q 20.8), los estudiantes que responden de modo afirmativo a esta pregunta hacen referencia a valores como la autenticidad, la veracidad y a la naturalidad.

Los participantes que contestan negativamente no dan razones, salvo una excepción que dice:

"No estoy cómoda porque muchas veces es falsa la imagen que doy en la red y presentamos exactamente lo que queremos que vean de nosotros"

Una estudiante explica que:

"Las críticas que se hacen en la red fomentan el odio y a veces nos provoca complejos de inferioridad".

Otro participante señala:

"Lo que se dice en las redes puede llevar a afectar seriamente a la salud psíquica de las personas." 
De igual manera, les afecta negativamente y les preocupa la gran obsesión por los cánones de belleza que se presentan en las RS. Otra de las participantes dice que pueden llevar incluso a algunos jóvenes a la depresión.

En segundo lugar, utilizan diversos términos para explicar la preocupación por no saber mantener la privacidad en las RS. Tienden a percibir el riesgo de perder la privacidad simplemente por "estar" en las redes.

Varios encuestados lo reflejan de esta manera:

"Estamos entregados a "depredadores" ya que tenemos toda nuestra información privada en las redes." "Tenemos cero privacidad".

Los jóvenes utilizan las palabras control y exposición de vida para referirse a la falta de intimidad personal. Conocen los riesgos existentes, como dice una de las participantes, de "subir todo a las redes".

En tercer lugar, destacan la ciberadicción o dependencia en el uso de las RS como la causa de la pérdida de amistades "presenciales" y de tiempo en general. Por ejemplo, una estudiante comenta: "Tengo mucha dependencia a mi teléfono y no disfruto de las cosas de alrededor."

Otros asuntos que les preocupan son el individualismo, la ignorancia sobre la utilización de la RS en general o la disminución de las vivencias del momento real.

\section{8. ¿Tiene una formación adecuada para entender los problemas vinculados con las redes? ( $Q$ 23.11)}

Más del 50\% de los encuestados contestan que tienen algún tipo de formación o que son autodidactas. Explican que conocen las "trampas" y los riesgos que tiene Internet. Otros estudiantes que contestan afirmativamente, hacen referencia al grado de experiencia en el uso de las redes, a la edad o la formación recibida en voluntariados, amistades, charlas, cursos o estudios universitarios. Un 16,64 \% contesta que es la familia la que les ha ayudado a entender y a usar correctamente las RS.

Otros estudiantes reflejan que es necesario adquirir nuevos conocimientos sobre todo en temas legales o sobre derechos personales. Uno de los participantes explica: "La tecnología avanza a gran ritmo, muchas veces más rápido de lo que podemos asimilar y se debe formar en su uso." "No tenemos las herramientas necesarias para controlar el volumen de información de la RS"

\section{9. ¿Qué formación académica has recibido para entender y usar las RS? (Q 25.13) y (Q 27.15)}

Un 34,56 \% de los jóvenes contestan que no les ha ayudado la formación académica. Uno de los participantes comenta: "No se dan instrucciones o simplemente no se enseña." No obstante, subrayan que se debería implementar alguna formación en el uso de las RS para "concienciar a los estudiantes."

Los que responden de modo afirmativo a la pregunta lo hacen porque han tenido alguna oportunidad de aprendizaje en la etapa de Educación Primaria o Secundaria. Otros universitarios 
han participado en cursos de formación, charlas o el tema de las RS se ha estudiado en alguna asignatura en la universidad.

\subsection{0. ¿Qué formación es necesaria para llegar a ser un miembro crítico en las redes sociales?}

El 78,08\% de los jóvenes ve necesario recibir algún tipo de formación para complementar la educación juvenil y explican que es importante prevenir a los adolescentes. Señalan que para llegar a ser un miembro crítico sería bueno:

1. Conocer el uso de las redes no solo a nivel tecnológico sino psicológico.

2. Aprender a gestionar las redes sociales personales.

3. Entender los peligros reales, saber reconocer las "fake news" y contrastar información.

4. Saber que es una fuente fiable.

5. Formarnos sobre temas relacionados sobre el impacto de la imagen personal en las redes.

6. Conocer los temas de protección de datos o posibles amenazas en la red.

Uno de los participantes comenta:

"Es necesario enseñar el uso correcto de las redes sociales, pero sin charlas inútiles ni asignaturas como tic. Es necesario una asignatura dedicada a la competencia digital bien estructurada."

Otros jóvenes no saben explicar qué tipo de formación digital se debería impartir o dicen, que se aprende con la experiencia o no lo ven necesario porque ya tienen formación.

Una de las participantes concluye que:

"Pienso que deberíamos aprender a comunicarnos mejor antes de comenzar a usar las redes sociales" y "La educación visual y mediática debería formar parte del currículo educativo y tener un mayor peso".

\section{Conclusión}

Hemos abordado el presente artículo planteando una cuestión inicial. ¿De qué modo puede afectar positiva o negativamente el uso que hacen los jóvenes universitarios de las redes sociales en su comprensión de la "participación ciudadana"? Esta pregunta es posible acometerla, dando respuesta a cada uno de los objetivos marcados.

En primer lugar, las características más valoradas por los jóvenes en el uso de las RS son: la inmediatez, la rapidez, la interconexión y la facilidad en su uso. La red más utilizada es WhatsApp seguida de Instagram, Facebook y YouTube. Las utilizan entre tres y cuatro horas al día.

En segundo lugar, frente al uso educativo, los universitarios/as utilizan principalmente las RS como canal de comunicación personal y con la pareja, para el entretenimiento y para organizar trabajos de clase. Las RS son una herramienta inmejorable para compartir lo que les interesa. En cuanto al ocio las utilizan para escuchar música, seguirla moda, la fotografía, el cine, el arte y los libros. También se sirven de las redes para descubrir destinos turísticos y organizar viajes. 
Los estudiantes consideran que, las redes son un medio excelente para influir positivamente en el modo de pensar, en las actitudes e intereses de los usuarios y para mejorar el mundo. Explican que las redes facilitan el compromiso ciudadano y una participación más activa en la sociedad por las posibilidades que ofrecen éstas de compartir ideas que favorezcan la reflexión, el debate, la concienciación social, estar al día o una buena convivencia social.

En tercer lugar, en cuanto a la participación social, el 69,05\% de los estudiantes tiene algún tipo de compromiso ciudadano y se considera un miembro responsable porque cumple con las normas, colabora en las campañas o firma propuestas online. El total de los estudiantes que expresan un compromiso en temas relacionados con el voluntariado y los que participan en varias ONG u otro tipo de asociaciones, es de un 30,17\%.

En cuarto lugar, los temas que les interesan más a los estudiantes son los relacionados con la educación y el medio ambiente. También destacan los contenidos que tienen relación con las desigualdades sociales, la equidad de género, los derechos humanos y las cuestiones raciales y culturales. Un tanto por ciento muy bajo está interesado en los partidos políticos y la salud.

Sin embargo, los jóvenes podrían dudar de la validez de las RS como canal apto de comunicación social para el desarrollo de la participación democrática. Reconocen los riesgos en el uso de la RS como son la anominia, la falta de privacidad y la existencia de información falsa. También consideran que es sencillo "subir" información errónea a internet y aluden a la ignorancia existente entre los internautas sobre determinados temas de tipo social o económico. Una de las participantes comenta: "La desinformación nos lleva a no saber qué pensar o a confundirnos".

Los estudiantes explican que las redes son un canal de comunicación que facilita la manipulación de los ciudadanos en el espacio virtual, bien difundiendo desinformación o mentiras que tras un anonimato virtual -como son los falsos perfiles- llevan a dudar de lo que es o no es verdad. Asimismo, entienden que la búsqueda exclusivamente de "likes" por parte de los usuarios, la utilización de las redes por los políticos con el fin de crear polémica o "promocionarse" o que las redes puedan ser controladas por los gobiernos, son riesgos que hacen de las redes un canal complejo para la participación social.

Los jóvenes aluden a la falta de ética en el uso en las RS utilizando los términos: el engaño, la crueldad, el ciberbullying, el uso de las redes para descalificar o desacreditar a personas, la competitividad, las faltas de respeto a las opiniones ajenas, juicios infundados, la existencia de contenidos "oscuros" o ambiguos.

No obstante, solo un $15,08 \%$ no participa en la mejora de su comunidad, y otro $15,08 \%$ se considera un miembro crítico al analizar las causas de los problemas y actuar sobre las mismas.

Finalmente, más del 50\% de los participantes consideran que están bien formados para entender los problemas existentes en la red, sin embargo, en las preguntas abiertas añaden que creen necesario una formación específica sobre el uso de las RS para llegar a ser miembros críticos en la sociedad. Más de la mitad de los encuestados contestan que es necesario recibir formación para complementar la educación juvenil, en el área cívico-digital. Insisten en que debemos prevenir a los adolescentes de los riesgos a nivel tecnológico y psicológico. 
Somos los docentes los que tenemos un gran reto por delante para ser los instrumentos que hagan de puente entre los jóvenes y las nuevas tecnologías. Los estudiantes demandan formación para saber gestionar las RS y desarrollar el pensamiento crítico. Incluir las redes sociales en el aula puede ser un buen modo de formarles en un uso responsable.

El análisis de la encuesta sobre el uso de las RS participación ciudadana y educación realizada en pleno confinamiento, nos ha confirmado que son los mismos problemas denunciados por los medios de comunicación y la ciudadanía en general, durante la primera oleada del COVID:19, los que han sido enumerados por los jóvenes en nuestro estudio: la falta de una ética cívico-digital, la irresponsabilidad o el uso incorrecto de las redes con la difusión de "bulos" y la preocupación social por mantener la privacidad personal.

Asimismo, son muy similares los beneficios experimentados y valorados por la sociedad en general durante la epidemia mundial, con los comentados por los jóvenes universitarios en la encuesta de nuestra investigación. Las redes e internet han solucionado y ayudado a sobrellevar muchas dificultades, pero sobre todo, las redes han acortado la distancia social con una cercanía virtual.

Como señalan los estudiantes en la encuesta, la inmediatez, la rapidez, la interconexión, la facilidad del uso de las RS y la ingente cantidad de información que se puede compartir en ellas, pero sobre todo las posibilidades de comunicación a nivel local y global, han sido las ventajas que nos han ofrecido las redes en estos momentos de crisis mundial.

Los beneficios derivados del uso de las RS que enumeran los jóvenes nos hacen concluir que a pesar de ser muchos y variados los riesgos que los participantes denuncian en el uso de las RS, también son muchas y variadas las posibilidades que ofrecen las RS como canal de participación ciudadana. Entendemos que es el espacio actual en el que la participación social juvenil puede crecer a nivel local y global.

\section{Limitaciones del estudio}

En primer lugar, es necesario aumentar la muestra para asegurar una distribución representativa de la población universitaria. En segundo lugar, al indagar lo que las personas opinan sobre el tema de estudio, los datos informados pueden contener potenciales sesgos. Finalmente, la muestra de la investigación corresponde en su mayoría a mujeres y el $90 \%$ de las/los participantes pertenecen a los grados de Educación Primaria e Infantil, por lo que se hace necesario ser cautos con las conclusiones presentadas en esta fase inicial de la investigación.

\section{Referencias bibliográficas}

Billieux, J., Maurage, P., Lopez-Fernandez, O., Kuss, D. J., \& Griffiths, M. D. (2015). Can disordered mobile phone use be considered a behavioral addiction? an update on current evidence and a comprehensive model for future research. Current Addiction Reports, 2, (2), 156-162. http://dx.doi.org/10.1007/s40429-015-0054-y

Bustamante Donas, J. (2007). Cooperación en el ciberespacio: Bases para una ciudadanía digital. Argumentos De Razón Técnica,10, 305-328. 
Calzada, I. (2017). Do Digital Social Networks Foster Civilian Participation among Millennials? Kitchenware Revolution and 15M Cases. Glocalism: Journal of Culture, Politics and Innovation 3. Special Issue: 'Beyond Democracy: Innovation as Politics'. http://dx.doi.org/10.12893/gjcpi.2017.3.4.

Castellanos Claramunt, J. (2019). Participación ciudadana en la era digital: Posibilidades y riesgos. Tesis Doctoral.

Castells, M. (2003). La dimensión cultural de internet. Nuevas tecnologías. Andalucía Educativa, 7, (36) Recuperado de https://www.uoc.edu/culturaxxi/esp/articles/castells0502/castells0502.html

Delgado-Algarra, E. J., Bernal-Bravo, C., \& López-Meneses, E. (2019). Competencia pluricultural y ciudadanía cosmopolita en el contexto hispano-japonés de educación superior. Educational Research, 8 (2), 166-183. http://dx.doi.org/10.7821/naer.2019.7.425

Fitzgerald, M., Kruschwitz, N., Bonnet, D., \& Welch, M. (2014). Embracing digital technology: A new strategic imperative. MIT Sloan Management Review, 55(2), 1.

Heater, D. (2007). Ciudadanía :Una breve historia. Madrid: Alianza.

Kymlicka, W., \& Norman, W. (2000). Citizenship in culturally diverse societies: Issues, contexts, $\begin{array}{lllll}\text { concepts. } \quad \text { Citizenship } & \text { in }\end{array}$ http://dx.doi.org/10.1093/019829770X.001.0001

Levinson, M. L. (2014). Citizenship and civic education. Encyclopedia of Educational Theory and Philosophy, ed Denis C. Phillips. Thousand Oaks, CA:age. Recuperado de http://nrs.harvard.edu/urn-3:HUL.InstRepos:12701475

Loader, B. D., Vromen, A., \& Xenos, M. A. (2014). The networked young citizen: Social media, political participation and civic engagement. Information, Communication \& Society, 17 (2), 143-150. http://dx.doi.org/10.1080/1369118X.2013.871571

Marín, H. (2019). Civismo y ciudadanía (2019) Madrid. Editorial La Huerta Grande.

Mata, J. C., Sabater, M. M., \& Blanch, J. P. (2019). Emociones y pensamiento crítico en la era digital: Un estudio con alumnado de formación inicial. REIDICS: Revista De Investigación En Didáctica De Las Ciencias Sociales, 5, 23-41.

Mercedes, G. V. M. (2015). Migración de los jóvenes españoles en redes sociales, de tuenti a facebook y de facebook a instagram. la segunda migración. ICONO 14, Revista De $\begin{array}{llllll}\text { Comunicación } & y & \text { Tecnologías } & \text { Emergentes, } & \text { 48-72. }\end{array}$ https://dx.doi.org/10.7195/ri14.v13i2.821

Naval, C. (2003). Orígenes recientes y temas clave de la educación para la ciudadanía democrática actual. Revista De Educación. Ministerio De Educación Y Formación Profesional. 9, 169-189.

Nelson, J., Lewis, D., \& Lei, R. (2017). Digital democracy in america: A look at civic engagement in an internet age. Journalism and Mass Communication Quarterly, 94, (1), 318-334. https://dx.doi.org/10.1177/1077699016681969

Nobles, D., Londoño, L., Martínez, S., Ramos, A., Santa, G., \& Cotes, A. (2015). Tecnologías de la comunicación y relaciones interpersonales en jóvenes universitarios. Educación y Humanismo, 18, (30) 14-27. https://doi.org/10.17081/eduhum.18.30.1311 
Padilla Castillo, G. (2013) Usos de las redes sociales entre los universitarios españoles como nuevo paradigma de comunicación. En Beatriz Lloves Sobrado; Francisco Segado Boj (dir. congr.) I Congreso Internacional de Comunicación y Sociedad Digital, 31.

Pennycook, G., McPhetres, J., Zhang, Y., \& Rand, D. (2020). Fighting COVID-19 misinformation on social media: Experimental evidence for a scalable accuracy nudge intervention. PsyArXiv Preprints, 10. https://doi.org/10.31234/osf.io/uhbk9

Cartes, D. (2020). La periodización y la conciencia histórica en la formación del profesorado. REIDICS: Revista De Investigación En Didáctica De Las Ciencias Sociales, 6, 6-23.

Ribble, M. S., Bailey, G. D., \& Ross, T. W. (2004). Digital citizenship: Addressing appropriate technology behavior. Learning \& Leading with Technology, 32(1), 6.

Rodríguez, A. E. G. (2008). Educación para la ciudadanía: Una aproximación al estado de la cuestión. Enseñanza De Las Ciencias Sociales: Revista De Investigación, 7, 131-140.

Sant, E. (2014). What does political participation mean to spanish students? JSSE-Journal of Social Science Education, 13, (4), 11-25. https://doi.org/10.2390/jsse-v13-i4-1321

Simsek, E., \& Simsek, A. (2013). New literacies for digital citizenship. Online Submission, 4 (3), 126137.

Zhang, C., Shi, L., \& Wang, F. (2020). Liver injury in COVID-19: Management and challenges. The Lancet. Gastroenterology \& Hepatology, 5, (5), 428-430. https://doi.org/10.1016/S24681253(20)30057-1 